\title{
The Experience of Nurses Providing Home Nursing Care to Oldest Old Persons Living Alone in Rural Areas-An Interview Study
}

\author{
Tove Mentsen Ness ${ }^{1,2}$, Ove Hellzen ${ }^{1}$, Ingela Enmarker ${ }^{1,2,3}$ \\ ${ }^{1}$ Department of Nursing, Mid-Sweden University, Östersund, Sweden \\ ${ }^{2}$ Department of Health Sciences, Nord-Trøndelag University College, Namsos, Norway \\ ${ }^{3}$ Centre of Care Research, Mid-Norway, Steinkjer, Norway \\ Email: tove.m.ness@hint.no
}

Received 18 March 2015; accepted 30 March 2015; published 2 April 2015

Copyright (C 2015 by authors and Scientific Research Publishing Inc.

This work is licensed under the Creative Commons Attribution International License (CC BY).

http://creativecommons.org/licenses/by/4.0/

(c) (i) Open Access

\section{Abstract}

The rapidly increasing population of older persons worldwide, and the fact that the majority of them want to continue living in their own homes, mean there is a growing focus on home based care. Because of this, it is necessary to increase the number of studies, including rural areas, as earlier studies are sparse. Rural areas cannot be seen as a homogeneous phenomenon, meaning more research is needed to increase knowledge about cultural differences in rural areas. The aim of this study was therefore to describe registered nurses' experiences of providing home nursing care to oldest old persons living alone in rural areas. A sample of 15 registered nurses in rural South Sami areas was chosen for this study, 13 women and 2 men. Narrative interviews were conducted, and qualitative content analysis was used to interpret the data. The analysis revealed four themes and eight subthemes in addition to a core-theme. The latent meaning of the themes "Feeling responsible", "Trying to accommodate", "Being challenged" and "Feeling significant" formed the core-theme: contradictions between nurses' ideals of being professional and the reality faced in rural home nursing care with close social relationships. The findings in this study showed that the experiences of providing home nursing care in rural areas to oldest old persons were multifaceted and altering, as well as emotionally and socially contradictory.

\section{Keywords}

Rural, Home Nursing Care, Nurses Experiences

\section{Introduction}

This study is the fourth paper in a larger study designed to illuminate the phenomena home nursing care in rural

How to cite this paper: Ness, T.M., Hellzen, O. and Enmarker, I. (2015) The Experience of Nurses Providing Home Nursing Care to Oldest Old Persons Living Alone in Rural Areas-An Interview Study. Open Journal of Nursing, 5, 336-344.

http://dx.doi.org/10.4236/ojn.2015.54036 
areas, as experienced by both care receivers and care providers. Elsewhere in this project, oldest old people describe their experiences. Findings in one sub-study show that nurses still use subtle colonialism techniques in their relationships with old south Sami people [1]. A second sub-study showed that being an oldest old man living alone in a rural area could be interpreted as being in a struggle between a dependent existence and a desire to be independent [2]. The third paper implied that being an oldest old woman living alone in a rural area meant living in the intersection between embracing the present in solitude and fearing the future with additional declining health [3].

Demographics are changing and a rapidly increasing population of older persons can be seen worldwide, of which the majority want to live in their own homes [4]. This means that disease structure also changes, as do medical care demands. With such structural changes comes an increasing need for an extended home nursing care. The organisation of home nursing care differs both within and between countries [5]. In recent years, due to advanced medical care many people experience either severe or mild frailty, throughout the elderly stage, which means there is a demand for medical care that also allows the recipient to have a good quality of life. For example, in Norway home nursing care during the last two decades has become the most important arena for municipal care services [6].

Decreasing health may contribute to isolation and loneliness for older people, and has implication to nurses in home nursing care. Therefore, when caring for older people, loneliness can represent a challenge to nurses, as they have to recognize symptoms and use appropriate interventions [7]. Reasonably, in rural areas older people have an increased risk for loneliness due to an increased risk for social isolation, due to the long distance between others. As an opportunity to maintain social and personal connection, connections with care providers are regarded as advantageous [8] and sharing non-clinical information may support a sense of connectedness and improve well-being to older persons living in rural areas and receiving home nursing care [9].

Providing nursing care in the home sometimes means clinical priorities to mind the gap between heavy workload and staff shortages [10]. This requires nurses to adapt to current situations that demand ethical maturity [11]. According to Gaut [12], caring is ethical in and of itself. Therefore, it is important that caring needs to recognize and focus on providing necessary, interpersonal, and intersubjective conditions for the development of a sense of security that people in need of nursing care seek and need. Person-centred care is an established tradition in nursing [13], which entails e.g. seeing the person behind the patient [14] and facilitating empowerment and shared decision-making [15]. This not only enhances improved quality of life for care receivers [16] [17], but it may also lead to better job satisfaction for care providers [18].

The fact that a majority of older people want to continue to live their lives, in their house and in their home community means there is a growing focus on home based care. With this in mind, it is necessary to increase the number of studies that focus on home nursing care in rural areas. When literature that focuses on home nursing care in rural areas is sparse, and rural areas are not seen as a homogeneous phenomenon, this means that more research is needed to increase the knowledge about cultural differences in rural areas. The aim of this study was therefore to describe the experiences of registered nurses providing home nursing care to oldest old persons living alone in rural areas.

\section{Method}

A qualitative design was chosen for this study. When studying peoples' experiences, lives, and worlds it seems important to talk to them with the purpose of trying to understand the world from their point of view [19]. This method applies an inductive style, where the participants' perspectives form the basis for new knowledge and the aim is not to generalize, but rather see the uniqueness in each situation [20].

\subsection{Context and Participants}

A sample of 15 registered nurses working in home nursing care in the rural South Sami area in Norway was chosen for this study. They lived and worked in four different municipalities. To gain access to the informants, health care leaders in each municipality were contacted. The health care leaders told the first author who wanted to participate in the study. Meetings were then arranged by the first author, but the informant decided time and place. The informants were informed verbally and by written information about the purpose of the study, both by the health care leader and by the first author. 


\subsection{Interviews}

Narrative interviews [21] were conducted by the first author from summer to autumn 2014, when nurses were asked to narrate stories about providing care to oldest old persons living alone in rural areas.

"Please, can you tell about your experiences of providing care to oldest old persons living alone in your municipality?”

Additionally, the nurses were asked to narrate stories about providing care to patients above 80 years old. During the interviews, clarifying questions were asked when the interviewer did not understand or if deeper reflections were needed. The interviews took place at the local nursing home in the municipality and lasted 43 105 minutes $(\mathrm{md}=59)$. The interviews were recorded and transcribed verbatim. All personal information was replaced with codes and these codes and transcriptions were kept at different locations at the university to ensure confidentiality.

\subsection{Data Analysis}

Content analysis is a method that systematically analyses written or verbal communication [22] and has been defined as "a process of identifying and categorizing the primary patterns in data" [19]. The analysis was done in the following way: each interview was read thoroughly several times and as opened minded as possible in order to get a sense of the whole. The text was then structurally analyzed and read to identify meaning units, guided by the aim of the study, and labelled with codes. The meaning units were then condensed into shorter formulations. The condensed and coded meaning units were analysed and compared with respect to differences and similarities, and subsequently abstracted to subthemes. Subthemes were then formulated for subsequent abstraction into themes, and, finally, an interpretation of the whole was made, resulting in an overall core-theme [19]. An example of the analysis process is given in Table 1.

To ensure trustworthiness in themes and subthemes, the analysis process involved back and forth movement between the whole text and its parts. The authors also discussed possible interpretations until consensus was reached [19].

\subsection{Ethical Considerations}

Informed consent was obtained before participation and information about the opportunity to withdraw from the study were given. Permission for research was granted by Social Science Data Services in Norway and accomplished in accordance to the Declaration of Helsinki [23].

\section{Results}

The sample of 15 registered nurses consisted of 13 women and 2 men. Their ages ranged from 28 to 56 (md =

Table 1. Example of the analysis process.

\begin{tabular}{|c|c|c|c|}
\hline Meaning unit & Condensed meaning unit & Subtheme & Theme \\
\hline $\begin{array}{l}\text { "It's natural for me as a nurse in the home nursing care and } \\
\text { change lightbulb if needed. I get it at the grocery store, and } \\
\text { change it the next time I' m there. I don't wait for the next } \\
\text { of kin to take care of it" }\end{array}$ & $\begin{array}{l}\text { Natural to change lightbulb as nurse. Get } \\
\text { it in the grocery. Change next visit. Do } \\
\text { not wait for next of kin. }\end{array}$ & Being attentive & $\begin{array}{l}\text { Feeling } \\
\text { responsible }\end{array}$ \\
\hline $\begin{array}{l}\text { "I use more time on those who are alone. If you know the } \\
\text { daughter will come soon, it is easier to leave. If you know } \\
\text { she is going to sit alone all day, you sit a little longer. Maybe } \\
\text { a chat or a cup of coffee" }\end{array}$ & $\begin{array}{l}\text { Use more time on those alone. Easer to } \\
\text { leave if relatives come. Sit alone all day, } \\
\text { stay longer }\end{array}$ & Being attentive & $\begin{array}{l}\text { Feeling } \\
\text { responsible }\end{array}$ \\
\hline $\begin{array}{l}\text { "It's awful to tell them that they have to go to bed so early, } \\
\text { because we cannot come later because of distance to our } \\
\text { office. Maybe they have to go to bed 1930, and we are not } \\
\text { there again before the next morning at 0900" }\end{array}$ & $\begin{array}{l}\text { Awful tell them go to bed 1930, because } \\
\text { of distance office. Back next day } 0900\end{array}$ & $\begin{array}{l}\text { Feeling } \\
\text { insufficient }\end{array}$ & $\begin{array}{l}\text { Being } \\
\text { challenged }\end{array}$ \\
\hline $\begin{array}{l}\text { "Some women do not want help from me when showering, } \\
\text { because I am a man, then we just change zone, so somebody } \\
\text { else can help that woman" }\end{array}$ & $\begin{array}{l}\text { Some women do not want help from men } \\
\text { when showering. Change zone. }\end{array}$ & Trying to adjust & $\begin{array}{l}\text { Trying to } \\
\text { accommodate }\end{array}$ \\
\hline
\end{tabular}


43). The nurses had worked between one to 34 years $(\mathrm{md}=13$ ), and work experience in home nursing care varied from one to 15 years $(\mathrm{md}=7)$.

Contradictions between nurses' ideals of being professional and the reality faced in rural home nursing care with close social relationships.

The analysis revealed a core-theme showing that nurses in home nursing care experience contradictions between their wish to care in a professional sense and to care in social sense. This core-theme is based on four themes and eight subthemes. The themes summarize nurses' views of providing home nursing care to oldest old persons living alone in rural areas. The emerging themes were "Feeling responsible", "Trying to accommodate", "Being challenged" and "Feeling significant".

\section{Theme 1: Feeling responsible}

The theme "feeling responsible" was built upon two subthemes: being attentive and utilizing time while providing care to oldest old persons in home nursing care.

Being attentive

To live and being a nurse in home nursing care in small rural communities sometimes means being in a different reality compared to those working in urban areas. Nurses often experience the need of being attentive because they often know the person for whom they are providing care. This implies that the oldest old person the nurse provides care for during her workday may be a colleagues' close family. This sometimes implicates the nurse and her work. One nurse said:

"We contribute more, because it can be the mother of someone we work with...//...We want to provide a little extra to people we know".

Nurses also expressed that they have to spend more attention to these oldest old people living alone who have no relatives or friends nearby. They experienced that these care receivers were harder to leave after finished achievements. For example, nurses expressed a need for more attention to these care receivers' health and requirements on themselves to insure the old person's nutrition state and medication before leaving. One nurse said:

"If I have given an old lady medication because of her diabetes, I get a little worried, I wonder if she is going to eat, does she remember? Maybe she is a little demented as well.”

Sometimes nurses admit that they go further than their professional obligation demands require, and give more attention when they see this necessary. For example, they do different practical chores such as carrying wood into the house or washing the kitchen floor, chores that usually a home-helper, relative, or neighbour does. Nurses also pay special attention to the oldest old persons who express that they feel lonely or those who do not demand anything from the home nursing care, so they can get more time to express possible needs. They also said they had to check more exhaustively if an old person gave the impression of not being at home when the nurse arrived to an agreed visit, e.g., by calling or paying an extra visit. Overall, nurses admit that receiving gratitude from the care receiver induced them to provide more care as a positive response entails willingness to become extra attentive to the oldest old persons.

Utilizing time

The nurses in home nursing care expressed that they want make good use of the time they have with the care recipient. They do this by working on several tasks at the same time, e.g., listening and commenting on important issues for the oldest old person while they perform wound care.

"You have to use your time well, and don't convey bustle. You have to concentrate those 5 minutes and be where you are."

They also said they were aware of their body language, and did not want to show through physical expressions that they are sometimes short of time when visiting a care receiver. Utilizing available time therefore is of great importance, which means sitting down, taking off their coat, and listening to the oldest old person those minutes they are present.

"It's important that you sit down, take of your coat and lay down your phone. It's there, but you can sit still."

\section{Theme 2: Trying to accommodate}

The theme "trying to accommodate" was built upon three subthemes: trying to adjust, trying to respect boundaries and trying to respect choices while providing care to oldest old persons in home nursing care.

Trying to adjust

The nurses tried to adapt to the oldest old persons in different ways. For example, adjustment to the care receiver's wish of not being showered by nurses of the opposite sex. The nurses also tried to adjust when something 
challenging or sensitive was to be discussed with the oldest old person.

"When you have challenging issues to discuss with the patient, it's important to consider which nurse who should talk with the patient, so it can be experienced as good as possible for the patient”

The nurses also try to adjust when the care receiver wants to stay in their home instead of moving to a nursing home despite their decreasing health. This can be done through increasing the amount of visits for a period of time or permanently. Some nurses enhance the importance of trying to adjust to the care receivers' culture. When they meet indigenous people, such as people with a South Sami background some nurses try to learn some South Sami words and learn about their culture. However, as one nurse said, they do not demand anything from us, "maybe they have adjusted to us and not the other way around?"

The oldest old persons generally had small demands towards home nursing care staff. Because of this, the nurses occasionally struggled to discover the oldest old person's wishes and needs for nursing. In addition, care receivers sometimes expressed that the nurse had done enough and that she/he might leave and help other care receivers.

Trying to respect boundaries

Nurses said they saw that the care receivers had different boundaries and therefore saw the importance of treating the oldest old persons in different ways when they got to know them, because they as care receivers have different boundaries. For example, this could entail different ways of entering an oldest old person's house and saying hello.

"I am aware of their boundaries. When I step over their doorstep I'm in their territory, and I am a guest even though I'm there to help."

The nurses expressed that they tried to respect the oldest old person's boundaries by being humble, and taking it slow when they meet them as new care receivers. This nursing strategy was aimed to getting to know the old person better and to find out what they needed to secure not overrunning them with their own meanings. Conversely, nurses enhance the difficulties of doing so occasionally, because they see the need to e.g. take a shower or change clothes. This means that they sometimes had to correct themselves after request from the oldest old person.

"I can be a little too persistent when it comes to showering, and then they can say so. That's good, because then I can correct myself."

Trying to respect choices

The nurses highlight that they sometimes meet care receivers who do not want to follow the nurses' recommendations and use their knowledge. Nurses usually try to respect the oldest old person's wishes, but they emphasize that it is a question of understanding the results of the choice. It is a question of medical matters; for example, in cases of a doctor visit, wound care, medications, or moving closer to a sheltered housing in the community centre professional decisions should prevail. One nurse said:

"Not everybody wants the service we offer, and we have to accept that, even if it's wound care or medications."

The nurses enhance using their professional knowledge when trying to convince the oldest old persons about what the nurse see as the best option for him or her. On such occasions, they stress the importance of trying to respect the person's own choices and not trying to persuade someone against his or her will for example, this can concern South Sami care receivers who may prioritize reindeer herding (following the reindeer heard up into the mountains) instead of focusing on wound care or medication.

"We can encourage change, but we cannot give orders to change."

\section{Theme 3: Being challenged}

The theme "being challenged" was built upon two subthemes: trying to treat everybody equal and feeling insufficient while providing care to oldest old persons in home nursing care.

Trying to treat everybody equal

Equality seems to be essential for the interviewed nurses. The nurses emphasized that they want to face all persons equally and that providing nursing is no different, regardless of age, gender, or cultural background.

"I have the same recipe for all. It's no difference on that...//... My nursing is the same to all. That's not even an issue."

Trying to treat everybody equal sometimes implies meeting persons that perceive situations differently from the nurses; this can be either care recipients or their relatives. Nurses said that older persons with a South Sami background, for example, sometimes have a different view on knowledge and time and that this cultural difference 
could involve complications in nurse-patient relationships. One nurse said:

"From my point of view they have a very irregular lifestyle, and a different diet, and that's not optimal if they have diabetes and a wound, and our suggestions are not followed. In the beginning when NN didn't do as I said, I wondered, have I explained myself badly?”

Similar situations sometimes happen when they meet relatives of the oldest old persons who live in other parts of the country. Sometimes they may question the nurse's knowledge, or want nurses to implement measures the nurses do not see as necessary. This challenges nurses, who sometimes feel they have more knowledge about the oldest old person than the relative does, because they see the person on a daily basis. The nurses said they try to treat all oldest old persons who live alone alike, by using extra time on them all, and not just those who request their presence.

“We try to have an even line, which is important, so one doesn't use all free time on the one that demands the most".

Trying to treat every care receiver equally can therefore become a challenging issue for the nurses as the care receivers themselves have different demands towards home nursing care.

Feeling insufficient

The nurses felt insufficient when they could not provide equal care to the oldest old persons in the municipality. Such feelings often depend on rural causes; for example, the distance to the municipality centre. Sometimes the oldest old person reject the offer of a sheltered housing because they want to stay in their own home. This may lead to feelings of insufficiency in the nurses because they feel that the oldest old person cannot receive the necessary care on such occasions.

"It's hard to leave, when you don't know what state they will be in when you return."

A feeling of insufficiency is also present when they cannot ease the oldest old person's feelings of loneliness.

"You feel discouraged sometimes when you don't manage to help them find something to fill their lives with, but you are not in charge of everything."

\section{Theme 4: Feeling significant}

The theme "feeling significant" was built upon one subtheme: feeling important while providing care to oldest old persons in home nursing care.

Feeling important

Gratitude and the positive response they described they experience from the oldest old persons makes them feel that they are important in someone's life. The gratitude the oldest old person shows them as nurses could be expressed in small gifts, for example a fruit or a cake but usually verbally or through a smile. All nurses said that the oldest old person's demands are very small or often non-existent. According to the nurses' experiences, this is especially common among those living alone. Being the peak of the day to some care recipients, and feeling important in someone's life enhances the feeling of significance. One nurse said:

"It is good to be the peak of the day for some, and mean something for those we meet."

Through their contribution to the oldest old person's well-being, nurses experience feelings of importance.

"It is meaningful when you see them light up when you come, and you notice that it is good for them. It is good to contribute to the patient's well-being."

The feeling of importance when providing care enhances a positive emotion for the nurses and contributes to a positive ambience in the patient-care provider relationship.

\section{Discussion}

The aim of this study was to describe the experience of nurses providing home nursing care to oldest old persons living alone in rural areas. The findings showed that the experiences of providing home nursing care were multifaceted and altering, as well as emotionally and socially contradictory. The latent meaning of these themes formed the core-theme: contradictions between nurses' ideals of being professional and the reality faced in rural home nursing care with close social relationships.

Caring for oldest old persons living alone in rural areas as experienced by nurses in home nursing care, involves an altruistic element implying a will to help and support them (e.g., [24]). The nurses may emanate from a conscious or unconscious desire to create as good a life as possible for the oldest old person, which may arise individuality for the patient [9]. This corresponds to the interpersonal dimension of caring; that is, to the fact that caring generates power within the relationships and when used appropriately it can help the patient evolve [25]. 
However, the nurses in the current study emphasized that they wanted to treat everybody equally regardless of gender, age, and cultural background. In that way, they may worsen the situation by instinctively trying to distance themselves from the patient through offering the same care to everyone instead of offering person centred care; i.e., to provide care by seeing the whole person with different beliefs and values [26]. Such distancing may lead to impaired interpersonal relations and may disturb the oldest old person's progress and quality of life [27].

The nurses in our study seemed to act intuitively on the basis of creating as much felicity as possible for the lonely oldest old persons. According to Nummela et al. [28], a decrease in the experience of loneliness increases perceived physical and mental health. This can be interpreted as when encountering, for example, indigenous people, such as oldest old persons with a South Sami background. The nurses must be courageous and acknowledge their own fear for other cultures. This may enrich their professional practice and enable nurses to see beyond their fear and act according to oldest old people's needs and desires [29].

Dahlberg [30] stated that voluntary loneliness could be a powerful and creative experience, which offered inner peace and calm. This corresponds to the opportunities offered by loneliness among the oldest old persons as living in confidence and feeling free [3] [31]. This means that the power that is inherent within the interpersonal relationship is a central determinant in the oldest old person's quality of life.

The inherent power within the interpersonal relationship may be militated through the organisation of the healthcare system as evidence based culture [32]. This implies that being a professional nurse in home nursing care means meeting oldest old people out of professional expectations of stringent evidence quality standards and medical classification systems. Consequently, the premise is that decisions, which are made on the basis of caring, do not meet the prerequisites in an evidence based culture (cf. [33]). Nurses' organisational attitudes, routines, and schedules seem to run counter to older people's wills [34]. Some nurses seem to consider their work from the organisation's perspective when making decisions on care, for example in this study when emphasizing giving the same care to all patients.

The contradictions between nurses' ideals of being professional and the reality faced in rural home nursing care with close social relationships involves ethically difficult situations. They may arise when nurses are not able to offer oldest old people care based on their needs or when they mistrust the patient's abilities. Concurrent to Kvåle and Bondevik [15], the nurses in our study tried to accommodate by respecting choices and boundaries, but the oldest old persons did not always want to receive the care the nurses meant to be the best option. From the older person's perspective, studies have shown that they experience that nurses' lack of respect towards their will and that their individual needs are not always met [35]. To solve this kind of ethical problem research indicates that knowing the oldest old person's life history is essential for nurses (e.g., [36]).

\subsection{Methodological Discussion}

The number of participants in this study is small. However, the conducted interviews resulted in a large amount of material, illuminating differences and similarities in the participants' experiences of providing home nursing care to oldest old persons living alone in rural areas. Thus, 15 participants were assessed to be enough, as trustworthiness in a qualitative study is gained more by the richness of each interview than by sample size [37].

Some of the results and quotations of this study may reflect not only the experiences of providing home nursing care to oldest old persons living alone in rural areas, but also the experiences of encountering older persons suffering from loneliness. It is not always possible to separate intertwined feelings from each other; that is, experiences of providing home nursing care to oldest old persons living alone in rural areas and meeting older persons who are experiencing loneliness who cannot always be isolated.

Our interpretation should be considered as one possible understanding of the experiences of loneliness among people with poor mental health. According to Krippendorff [22], a text never implies one single meaning, just the most probable meaning from a particular perspective. In order to enhance trustworthiness, the authors discussed every step in the analysis process until consensus about the interpretation was achieved. We also reflected on our findings in relation to the text and relevant literature.

\subsection{Conclusion and Relevance for Practice}

Nurses' cultural competences and moral behaviour are most important in meeting with the oldest old persons. It plays a dominant role in the encounter and requires flexibility and respect towards the person's will and desire. More consideration is therefore essential for how nurses encounter the oldest old people with different needs of 
care in their own homes. To achieve this, continuous discussions and supervisions in work place are needed.

\section{Acknowledgements}

We would like to thank the participants who shared their stories with us.

\section{References}

[1] Ness, T.M., Enmarker, I. and Hellzen, O. (2013) Experiences of Being Old and Receiving Home Nursing Care. Older South Sami narrations of Their Experiences-An Interview Study. Open Journal of Nursing, 3, 1-7. http://dx.doi.org/10.4236/ojn.2013.31001

[2] Ness, T.M., Hellzen, O. and Enmarker, I. (2014) "Struggling for Independence”: The Meaning of Being an Oldest Old Man in a Rural Area. Interpretation of Oldest Old Men’s Narrations. International Journal of Qualitative Studies on health and Well-Being, 9, Article ID: 23088. http://dx.doi.org/10.3402/qhw.v9.23088

[3] Ness, T.M., Hellzen, O. and Enmarker, I. (2014) "Embracing the Present and Fearing the Future”: The Meaning of Being an Oldest Old Woman in a Rural Area. International Journal of Qualitative Studies on Health and Well-Being, 9, Article ID: 25217. http://dx.doi.org/10.3402/qhw.v9.25217

[4] Chandler, J., Williams, M., Maconachie, M., Collett, T. and Dodgeon, B. (2004) Living Alone: Its Place in Household Formation and Change. Sociological Research Online, 9. http://www.socresonline.org.uk/9/3/chandler.html

[5] Genet, N., Boerma, W.G.W., Kringos, D.S., Francke, A.L., Fagerström, C., Melchiorre, M.G., Greco, C. and Deville, W. (2011) Home Care in Europe: A Systematic Literature Review. BMC Health Services Research, 11, 207. http://www.biomedcentral.com/1472-6963/11/207

[6] Thygesen, E., Saevareid, H.I., Lindstrom, T.C. and Engdal, K. (2009) Psychological Distress and Its Correlates in Older Care-Dependent Persons Living at Home. Aging \& Mental Health, 13, 319-327. http://dx.doi.org/10.1080/13607860802534591

[7] Murphy, F. (2006) Loneliness: A Challenge for Nurses Caring for Older People. Gerontological Care and Practice, 18, 22-25. http://dx.doi.org/10.7748/nop2006.06.18.5.22.c2424

[8] Farmer, J., Philips, L., King, G., Farrington, J. and MacLeod, M. (2010) Territorial Tensions: Misaligned Management and Community Perspectives on Health Services for Older People in Remote Rural Areas. Health \& Place, 16, 275283. http://dx.doi.org/10.1016/j.healthplace.2009.10.010

[9] Corbett, S. and Williams, F. (2014) Striking a Professional Balance: Interactions between Nurses and Their Older Rural Patients. British Journal of Community Nursing, 19, 162-167. http://dx.doi.org/10.12968/bjcn.2014.19.4.162

[10] Tønnesen, S., Nordtvedt, P. and Førde, R. (2011) Rationing Home-Based Nursing Care: Professional Ethical Implications. Nursing Ethics, 18, 386-396. http://dx.doi.org/10.1177/0969733011398099

[11] Öresland, S., Määttä, S., Nordberg, A. and Lützen, K. (2011) Home-Based Nursing: An Endless Journey. Nursing Ethics, 18, 408-417. http://dx.doi.org/10.1177/0969733011398098

[12] Gaut, D.A. (1993) Caring: A Vision of Wholeness for Nursing. Journal of Holistic Nursing, 11, 164-171. http://dx.doi.org/10.1177/089801019301100205

[13] McCormack, B., Karlsson, B., Dewing, J. and Lerdal, A. (2010) Exploring Person-Centredness: A Qualitative Meta-Synthesis of Four Studies. Scandinavian Journal of Caring Sciences, 24, 620-634. http://dx.doi.org/10.1111/j.1471-6712.2010.00814.x

[14] Clarke, A., Hanson, E.J. and Ross, H. (2003) Seeing the Person behind the Patient: Enhancing the Care of Older People Using a Biographical Approach. Journal of Clinical Nursing, 12, 697-706. http://dx.doi.org/10.1046/j.1365-2702.2003.00784.x

[15] Kvåle, K. and Bondevik, M. (2008) What Is Important for Patient Centred Care? A Qualitative Study about the Perception of Patients with Cancer. Scandinavian Journal of Caring Sciences, 22, 582-589. http://dx.doi.org/10.1111/j.1471-6712.2007.00579.x

[16] Sjögren, K., Lindkvist, M., Sandman, P.O., Zingmark, K. and Edvardsson, D. (2013) Person-Centredness and Its Association with Resident Well-Being in Dementia Care Units. Journal of Advanced Nursing, 69, 2196-2206. http://dx.doi.org/10.1111/jan.12085

[17] Edvardsson, D., Petersson, L., Sjögren, K., Lindkvist, M. and Sandman, P.O. (2013) Everyday Activities for People with Dementia in Residential Aged Care: Associations with Person-Centredness and Quality of Life. International Journal of Older People Nursing, 9, 269-276. http://dx.doi.org/10.1111/opn.12030

[18] Lehuluante, A., Nilsson, A. and Edvardsson, D. (2012) The Influence of a Person-Centred Psychosocial Unit Climate on Satisfaction with Care and Work. Journal of Nursing Management, 20, 319-325.

http://dx.doi.org/10.1111/j.1365-2834.2011.01286.x 
[19] Patton, M.Q. (2002) Qualitative Research and Evaluation Methods. 3rd Edition, Sage, London.

[20] Creswell, J.W. (2009) Research Design: Qualitative, Quantitative, and Mixed Methods Approaches. 3rd Edition, Sage, London.

[21] Mishler, G. (1986) Research Interviewing: Context and Narrative. Harvard University Press, Cambridge.

[22] Krippendorff, K. (2013) Content Analysis_-An Introduction to Its Methodology. 3rd Edition, Sage, Thousand Oaks.

[23] World Medical Association (2008) WMA Declaration of Helsinki-Ethical Principles for Medical Research Involves Human Subjects. http://www.wma.net/en/30publications/10policies/b3/

[24] Heaslip, V. (2013) District Nursing: The Hidden Giant of the NHS? British Journal of Community Nursing, 18, 404406. http://dx.doi.org/10.12968/bjcn.2013.18.8.404

[25] Dunphy, L.M. and Winland-Brown, J.E. (1998) The Circle of Caring: A Transformative Model of Advanced Practice Nursing. Clinical Excellence for Nurse Practitioners, 2, 241-247.

[26] McCormack, B. and McCance, T. (2010) Person-Centred Nursing Theory, Models and Methods. Blackwell Publishing, Oxford. http://dx.doi.org/10.1002/9781444390506

[27] Hellzen, O., Asplund, K., Sandman, P.O. and Nordberg, A. (2004) The Meaning of Caring as Described by Nurses Caring for a Person Who Acts Provokingly: An Interview Study. Scandinavian Journal of Caring Sciences, 18, 3-11. http://dx.doi.org/10.1111/j.1471-6712.2004.00256.x

[28] Nummela, O., Seppänen, M. and Uutela, A. (2010) The Effect of Loneliness and Change in Loneliness on Self-Rated Health (SRH): A Longitudinal Study among Aging People. Archives of Gerontology and Geriatrics, 53, 163-167. http://dx.doi.org/10.1016/j.archger.2010.10.023

[29] Lindh, I.-B., da Silva, A.B., Berg, A. and Severinsson, E. (2010) Courage and Nursing Practice: A Theoretical Analysis. Nursing Ethics, 17, 551-565. http://dx.doi.org/10.1177/0969733010369475

[30] Dahlberg, K. (2007) The Enigmatic Phenomenon of Loneliness. International Journal of Qualitative Studies on Health and Well-Being, 2, 195-207. http://dx.doi.org/10.1080/17482620701626117

[31] Graneheim, U.H. and Lundman, B. (2009) Experiences of Loneliness among the Very Old: The Umeå 85+ Project. Aging and Mental Health, 14, 433-438. http://dx.doi.org/10.1080/13607860903586078

[32] Fagerström, L. and Engberg, I.B. (1998) Measuring the Unmeasurable: A Caring Science Perspective on Patient Classification. Journal of Nursing Management, 6, 165-172. http://dx.doi.org/10.1046/j.1365-2834.1998.00062.x

[33] Devik, S.A., Enmarker, I. and Hellzen, O. (2013) When Expressions Make Impressions-Nurses' Narratives about Meeting Severely Ill Patients in Home Nursing Care: A Phenomenological-Hermeneutic Approach to Understanding. International Journal of Qualitative Studies on Health and Well-Being, 8, 21880. http://dx.doi.org/10.3402/qhw.v8i0.21880

[34] Bolmsjö, I.Å., Sandman, L. and Andersson, E. (2006) Everyday Ethics in the Care of Elderly People. Nursing Ethics, 13, 249-263. http://dx.doi.org/10.1191/0969733006ne875oa

[35] Hellström, U.W. and Sarvimäki, A. (2007) Experiences of Self-Determination by Older Persons Living in Sheltered Housing. Nursing Ethics, 14, 413-424. http://dx.doi.org/10.1177/0969733007075888

[36] Dragset, I., Normann, K. and Elstad, I. (2015) Identity-Supportive Care of Patients with Dementia in Nursing Homes. Nordisk Sygeplejeforskning, in Press.

[37] Sandelowski, M. (1995) Focus on Qualitative Methods. Sample Size in Qualitative Research. Research in Nursing and Health, 18, 179-183. 